\title{
Prevalencia y factores de riesgo asociados a la infección por Cryptosporidium spp. en becerras lactantes en Aguascalientes, México
}

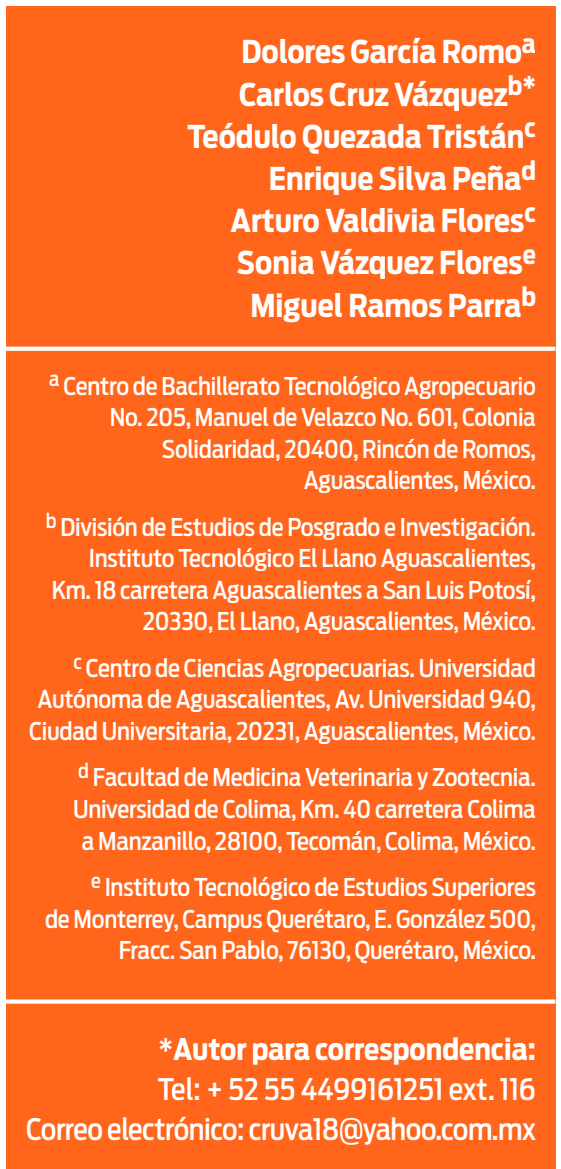

Recibido: 2014-03-26 Aceptado: 2014-09-17

Publicado: 2014-09-29

Información y declaraciones adicionales en la página 9

() Derechos de autor: Dolores García Romo et al. 2014

acceso abierto $\mathbf{\odot}$

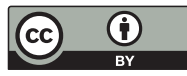

Distribuido bajo una Licencia Creative Commons Atribución 4.0 Internacional (CC-BY 4.0)

\section{Resumen}

El objetivo fue identificar la prevalencia y algunos factores de riesgo asociados a la infección por Cryptosporidium spp. en becerras lactantes mantenidas en confinamiento en tres establos lecheros de Aguascalientes, México. Durante 12 meses se recolectaron 1658 muestras fecales de becerras de 0 a 28 días de edad, que se procesaron mediante frotis fecal teñido con Kinyoun para identificar la presencia de ooquistes del parásito. Se aplicó una encuesta para identificar diferentes características de manejo en los establos y se desarrolló el análisis de riesgos mediante regresión logística. La prevalencia general a la infección por Cryptosporidium spp. fue de 40\%. El grupo de becerras de 8 a 14 días de edad presentó la prevalencia a la infección más elevada (81\%), mientras que la más baja (21\%) se observó en el grupo de 22 a 28 días de edad. Se identificaron como factores de riesgo a la infección por Cryptosporidium spp., al grupo de 8 a 14 días de edad $(\mathrm{OR}=2.5 ; 95 \% \mathrm{Cl} 1.9$ - 3.2, $\mathrm{p}<0.000)$; el uso de cama de aserrín $(\mathrm{OR}=1.8 ; 95 \% \mathrm{Cl} 1.1-2.8, \mathrm{p}<0.011)$; el recipiente de plástico de donde beben leche las becerras $(\mathrm{OR}=1.4 ; 95 \% \mathrm{Cl} 1.1-1.7, \mathrm{p}<0.000)$; el bajo nivel de inmunoglobulinas ( $\mathrm{OR}=1.8 ; 95 \% \mathrm{Cl} 1.1-2.9, \mathrm{p}<0.009)$, y la atención de partos por parte de personal no especializado (velador: $\mathrm{OR}=2.5$; $95 \% \mathrm{Cl} 1.6$ - 3.9, $\mathrm{p}<0.000$; trabajador del área de recría: $\mathrm{OR}=1.7 ; 95 \%$ Cl $1.2-2.5, p<0.001)$.

Palabras clave: Cryptosporidium spp; Ganado lechero; Becerras lactantes; Prevalencia; Factores de riesgo.

\section{Introducción}

Cryptosporidium spp. son parásitos protozoarios causantes de infecciones gastrointestinales en una amplia variedad de huéspedes vertebrados, incluidos animales domésticos y silvestres así como los humanos (Ramírez et al., 2004; Cacciò y Pozio, 2006; Ng et al., 2012). En el ganado bovino se han reconocido al menos cuatro especies, que incluyen a C. parvum, C. andersoni, C. bovis y C. ryanae. La primera de ellas es la más frecuente en animales lactantes, mientras que las otras son más comunes en animales destetados y adultos (Lindsay et al., 2000; 
Fayer et al., 2005, 2007, 2008). La criptosporidiosis es especialmente importante en animales lactantes menores de 30 días de edad, en quienes se manifiesta, por lo general, con diarrea abundante, fiebre, anorexia, pérdida de peso, desequilibrio electrolítico y, en ocasiones, con la muerte (Ramírez et al., 2004; Cacciò y Pozio, 2006). El grupo de mayor riesgo para contraer la infección es el de los becerros de 8 a 14 días de edad (Castro-Hermida et al., 2002a; Santín et al., 2004; Feitosa et al., 2004; Trotz-Williams et al., 2005), ya que al aumentar la edad de los animales, la prevalencia y la intensidad de la parasitosis tienden a disminuir (Castro-Hermida et al., 2002b; Ortolani y Soares, 2003; Starkey et al., 2006; Castro-Hermida et al., 2006; Bhat et al., 2013). Sin embargo, la infección es poco común en animales menores de cuatro días (Uga et al., 2000).

Los animales infectados con Cryptosporidium spp. pueden excretar por las heces un elevado número de ooquistes, los cuales se diseminarían fácilmente en el medio, de tal forma que diferentes factores de manejo han sido asociados a la infección por Cryptosporidium spp. Sería importante que estos factores se consideraran en programas de prevención de esta parasitosis, pues no se conocen tratamientos farmacológicos efectivos (Cacciò y Pozio, 2006). Sin embargo, algunos de estos factores varían según la región y los sistemas de producción, mientras que otros pueden estar presentes en cualquier establo. Entre los factores que se citan con mayor frecuencia se encuentran la edad, áreas de partos múltiples, la escasa frecuencia de aseo en las instalaciones, el material o tipo de cama, el uso de agua contaminada para consumo del ganado, el sistema de producción tecnificado, los corrales con piso de arena y la época de lluvias, entre otros (Maldonado et al., 1998; De la Fuente et al., 1999; Mohammed et al., 1999; Castro-Hermida et al., 2002b; Trotz-Williams et al., 2007; Brook et al., 2008; Almeida et al., 2010; Muhid et al., 2011 ; Silverlås y Blanco-Penedo, 2013; Bhat et al., 2013).

En México, se ha reportado la criptosporidiosis bovina en diversas regiones ganaderas (Maldonado et al., 1998; Castillo et al., 2009; Castelan-Hernández et al., 2011 ; Cano-Romero et al., 2011), y aún quedan numerosos tópicos por investigar acerca de su epidemiología en los diferentes sistemas de producción.

El objetivo fue identificar la prevalencia y algunos factores de riesgo asociados a la infección por Cryptosporidium spp. en becerras lactantes mantenidas en confinamiento en tres establos lecheros de Aguascalientes, México.

\section{Materiales y métodos Sitio de estudio}

Este análisis se llevó a cabo en el estado de Aguascalientes, localizado en la región centro-norte de México, a una altitud promedio de 1885 msnm. Este estado cuenta con una temperatura media anual de $16^{\circ} \mathrm{C}$ y una precipitación pluvial promedio de $475 \mathrm{~mm}$ al año, principalmente en el verano.

\section{Establos}

Por conveniencia se seleccionaron 3 establos, dos de ellos localizados al centro y norte del municipio de Pabellón de Arteaga, y otro al oriente del municipio de Rincón de Romos. En los 3 se contaba con ganado Holstein. Cada establo tenía un 
área de recría de becerras y realizaban prácticas de manejo y alimentación similares; asimismo, en los 3 las becerras lactantes se mantenían alojadas en salas de crianza con piso de concreto.

\section{Muestreo}

Se visitaron los establos cada 2 semanas durante un año para colectar una muestra fecal directamente del recto de todas las becerras entre 0 y 28 días de edad presentes el día de la visita, sin considerar si tenían diarrea. Cada muestra se identificó individualmente con la fecha de nacimiento. Ningún animal se incluyó dos veces en el estudio.

Adicionalmente, se recolectaron muestras de sangre por venopunción con equipo vacutainer nuevo en las becerras de 1 a 7 días de edad. Todas las muestras se mantuvieron en refrigeración para su traslado. En el laboratorio, se centrifugaron a 2500 rpm durante 15 minutos para obtener el suero, el cual se depositó en viales de polipropileno y se conservó a $-20^{\circ} \mathrm{C}$ hasta su uso. Debido a las características del estudio, no se requirió de la aprobación del Comité de Uso y Cuidado de Animales.

Con la finalidad de conocer la distribución de la parasitosis en la población estudiada, ésta última se clasificó en cuatro grupos: 0 a 7, 8 a 14, 15 a 21 y 22 a 28 días de edad.

\section{Diagnóstico coprológico}

Las muestras fecales se procesaron mediante frotis fecal teñido con Kinyoun para identificar la presencia de ooquistes del parásito. Por cada muestra se elaboró una laminilla con 6 frotis, que después de teñirse se observaron al microscopio. Para minimizar las lecturas de falsos positivos, se consideró una muestra como positiva cuando, después de examinar por completo los 6 frotis, se hubieran observado $>5$ ooquistes (Castillo et al., 2009).

\section{Determinación de la cantidad total de inmunoglobulinas en suero}

Para establecer la cantidad de inmunoglobulinas transferidas por el calostro a las becerras dentro de los primeros 7 días de vida, se utilizó la prueba de turbidez con sulfato de zinc. El número de unidades de turbidez del sulfato de zinc (UTSZ) corresponde a los miligramos de inmunoglobulinas totales por mililitro de suero, de manera que valores menores de 10 UTSZ indican una falla en la transferencia pasiva de anticuerpos de la madre a la cría; un rango de 10 a 20 representa un nivel aceptable, pero insuficiente, y más de 20 se considera como el nivel mínimo necesario para lograr una lactación exitosa en el neonato (Martínez y Ortega, 2011).

\section{Encuesta}

Para determinar los factores de riesgo hipotéticos asociados a la infección por Cryptosporidium spp., se usaron los datos obtenidos de una encuesta aplicada a los responsables de las áreas de crianza en los establos bajo estudio. Esta encuesta incluyó preguntas acerca de las características de manejo e instalaciones. Con los factores de riesgo hipotéticos se formaron 5 grupos, cada uno integrado con diferentes variables y sus respectivas categorías, los cuales se utilizaron como base para el análisis de riesgos (Cuadro 1). 
Cuadro 1. Prevalencia de la infección por Cryptosporidium spp. para las diferentes categorías en cada variable y grupo de características bajo estudio.

\begin{tabular}{|c|c|c|c|c|}
\hline Variable & Categorias & $n$ & Positivas & Prevalencia (\%) \\
\hline \multicolumn{5}{|c|}{ Grupo de características individuales de las becerras y sus madres } \\
\hline Edad & $\begin{array}{l}0 \text { a } 7 \text { días } \\
8 \text { a } 14 \text { días } \\
15 \text { a } 21 \text { días } \\
22 \text { a } 28 \text { días }\end{array}$ & $\begin{array}{l}506 \\
399 \\
408 \\
345\end{array}$ & $\begin{array}{c}107 \\
324 \\
164 \\
74\end{array}$ & $\begin{array}{l}33.5 \\
81.2 \\
40.1 \\
21.4\end{array}$ \\
\hline Tipo de heces & $\begin{array}{l}\text { Normales } \\
\text { Diarreicas } \\
\text { Semilíquidas }\end{array}$ & $\begin{array}{c}1362 \\
246 \\
50\end{array}$ & $\begin{array}{l}501 \\
144 \\
24\end{array}$ & $\begin{array}{l}36.7 \\
58.5 \\
48.0\end{array}$ \\
\hline Tipo de parto & $\begin{array}{l}\text { Distócico } \\
\text { Normal } \\
\text { No determinado }\end{array}$ & $\begin{array}{c}284 \\
1324 \\
50\end{array}$ & $\begin{array}{l}108 \\
541 \\
20\end{array}$ & $\begin{array}{l}38.0 \\
40.8 \\
40.0\end{array}$ \\
\hline No. de parto & $\begin{array}{l}1^{\circ} \text { a } 4^{\circ} \\
5^{\circ} \text { ó más }\end{array}$ & $\begin{array}{c}1509 \\
149\end{array}$ & $\begin{array}{c}604 \\
65\end{array}$ & $\begin{array}{l}40.0 \\
43.6\end{array}$ \\
\hline \multicolumn{5}{|c|}{ Grupo de características del manejo en el nacimiento y lactancia } \\
\hline $\begin{array}{l}\text { Persona que atiende } \\
\text { el nacimiento de la cría }\end{array}$ & $\begin{array}{l}\text { El velador } \\
\text { El trabajador del área de recría } \\
\text { El técnico de guardia }\end{array}$ & $\begin{array}{l}395 \\
512 \\
751\end{array}$ & $\begin{array}{l}145 \\
219 \\
266\end{array}$ & $\begin{array}{l}36.7 \\
42.7 \\
35.4\end{array}$ \\
\hline $\begin{array}{l}\text { Tipo de calostro consumido por } \\
\text { las crías }\end{array}$ & $\begin{array}{l}\text { Fresco } \\
\text { Refrigerado } \\
\text { No determinado }\end{array}$ & $\begin{array}{c}1328 \\
282 \\
48\end{array}$ & $\begin{array}{c}553 \\
12 \\
104\end{array}$ & $\begin{array}{l}41.6 \\
36.8 \\
25.0\end{array}$ \\
\hline Tratamiento anti- diarreico & $\begin{array}{l}\text { Sin tratamiento } \\
\text { Con tratamiento } \\
\text { No especificado }\end{array}$ & $\begin{array}{l}608 \\
169 \\
881\end{array}$ & $\begin{array}{l}257 \\
79 \\
333\end{array}$ & $\begin{array}{l}42.2 \\
46.7 \\
37.7\end{array}$ \\
\hline $\begin{array}{l}\text { Vacuna contra enfermedades } \\
\text { diarreicas }\end{array}$ & $\begin{array}{l}\text { No vacuna } \\
\text { Sí vacuna }\end{array}$ & $\begin{array}{l}751 \\
907\end{array}$ & $\begin{array}{l}266 \\
403\end{array}$ & $\begin{array}{l}35.4 \\
44.4\end{array}$ \\
\hline Nivel de inmunoglobulinas & $\begin{array}{l}\text { Aceptable } \\
\text { Medio } \\
\text { Bajo }\end{array}$ & $\begin{array}{c}12 \\
113 \\
202\end{array}$ & $\begin{array}{c}0 \\
21 \\
42\end{array}$ & $\begin{array}{r}0.0 \\
18.5 \\
20.7\end{array}$ \\
\hline \multicolumn{5}{|c|}{ Grupo de caracteristicas sobre la higiene de equipo e instalaciones } \\
\hline $\begin{array}{l}\text { Tipo e higiene de la cama y/o } \\
\text { piso del alojamiento }\end{array}$ & $\begin{array}{l}\text { De aserrín con cambio cada } 5 \text { días } \\
\text { De caucho con retiro de excremento a } \\
\text { diario y lavado semanal } \\
\text { Sin cama, piso tipo reja con retiro de } \\
\text { excremento a diario }\end{array}$ & 108 & $\begin{array}{l}39 \\
485\end{array}$ & $\begin{array}{l}36.1 \\
38.4\end{array}$ \\
\hline $\begin{array}{l}\text { Lavado y desinfección del } \\
\text { alojamiento individual }\end{array}$ & $\begin{array}{l}\text { Al desalojar, se lava y se aplica cal } \\
\text { Al desalojar se lava con sales cuaternarias } \\
\text { de amonio } \\
\text { Al desalojar se lava con cloro }\end{array}$ & $\begin{array}{l}395 \\
512 \\
751\end{array}$ & $\begin{array}{l}184 \\
219 \\
266\end{array}$ & $\begin{array}{l}46.5 \\
42.7 \\
35.4\end{array}$ \\
\hline $\begin{array}{l}\text { Frecuencia y forma de lavado } \\
\text { del recipiente para tomar leche } \\
\text { o sustituto de leche }\end{array}$ & $\begin{array}{l}\text { A diario con agua más detergente } \\
\text { A diario con agua y jabón yodado } \\
\text { A diario con agua. Con jabón yodado cada } \\
\text { tercer día }\end{array}$ & $\begin{array}{l}395 \\
512\end{array}$ & $\begin{array}{l}184 \\
219\end{array}$ & $\begin{array}{l}46.5 \\
42.7\end{array}$ \\
\hline
\end{tabular}




\section{Cuadro 1. Continuación}

\begin{tabular}{|c|c|c|c|c|}
\hline Variable & Categorías & $n$ & Positivas & Prevalencia (\%) \\
\hline $\begin{array}{l}\text { Lavado de biberones } \\
\text { para suministro de calostro }\end{array}$ & $\begin{array}{l}\text { Enjuague después de su uso } \\
\text { entre cada cría } \\
\text { Con jabón más iodo después de su uso } \\
\text { entre cada cría }\end{array}$ & $\begin{array}{l}1573 \\
85\end{array}$ & $\begin{array}{l}642 \\
27\end{array}$ & $\begin{array}{l}40.8 \\
31.7\end{array}$ \\
\hline $\begin{array}{l}\text { Frecuencia y forma de lavado } \\
\text { del piso de la sala de crianza }\end{array}$ & $\begin{array}{l}\text { Semanal con agua } \\
\text { A diario con agua } \\
\text { A diario con jabón yodado }\end{array}$ & $\begin{array}{l}395 \\
427 \\
836\end{array}$ & $\begin{array}{l}184 \\
192 \\
293\end{array}$ & $\begin{array}{l}46.5 \\
44.9 \\
35.0\end{array}$ \\
\hline \multicolumn{5}{|c|}{ Grupo de caracteristicas sobre el tipo de equipo e instalaciones } \\
\hline Tipo de sala de crianza & $\begin{array}{l}\text { Cerrada } \\
\text { Semicerrada } \\
\text { Abierta }\end{array}$ & $\begin{array}{l}1037 \\
395 \\
226\end{array}$ & $\begin{array}{c}395 \\
184 \\
90\end{array}$ & $\begin{array}{l}38.0 \\
46.5 \\
39.8\end{array}$ \\
\hline Alojamiento individual & $\begin{array}{l}\text { Corraleta } \\
\text { Metálica elevada }\end{array}$ & $\begin{array}{c}395 \\
1263\end{array}$ & $\begin{array}{l}184 \\
485\end{array}$ & $\begin{array}{l}46.5 \\
38.4\end{array}$ \\
\hline $\begin{array}{l}\text { Tipo de recipiente para tomar } \\
\text { leche o sustituto de leche }\end{array}$ & $\begin{array}{l}\text { Plástico } \\
\text { Lámina galvanizada } \\
\text { Los dos anteriores }\end{array}$ & $\begin{array}{l}907 \\
226 \\
525\end{array}$ & $\begin{array}{c}403 \\
90 \\
176\end{array}$ & $\begin{array}{l}44.4 \\
39.8 \\
33.5\end{array}$ \\
\hline \multicolumn{5}{|c|}{ Grupo de características de la operación y administración del establo } \\
\hline Tamaño del hato & $\begin{array}{l}\leq 1000 \text { cabezas } \\
>1000 \text { cabezas }\end{array}$ & $\begin{array}{c}395 \\
1263\end{array}$ & $\begin{array}{l}184 \\
485\end{array}$ & $\begin{array}{l}46.5 \\
38.4\end{array}$ \\
\hline $\begin{array}{l}\text { Otras especies animales en el } \\
\text { establo }\end{array}$ & $\begin{array}{l}\text { No hay } \\
\text { Sí hay }\end{array}$ & $\begin{array}{l}1057 \\
601\end{array}$ & $\begin{array}{l}413 \\
256\end{array}$ & $\begin{array}{l}39.0 \\
42.5\end{array}$ \\
\hline $\begin{array}{l}\text { Edad de los operarios del área } \\
\text { de recría }\end{array}$ & $\begin{array}{l}\leq 20 \text { años } \\
\text { De } 21 \text { a } 30 \text { años } \\
>31 \text { años }\end{array}$ & $\begin{array}{l}226 \\
920 \\
512\end{array}$ & $\begin{array}{c}90 \\
360 \\
219\end{array}$ & $\begin{array}{l}39.8 \\
39.1 \\
42.7\end{array}$ \\
\hline Incentivos a los operarios & $\begin{array}{l}\text { No otorgan } \\
\text { Sí otorgan }\end{array}$ & $\begin{array}{l}907 \\
751\end{array}$ & $\begin{array}{l}403 \\
266\end{array}$ & $\begin{array}{l}44.4 \\
35.4\end{array}$ \\
\hline Origen del ganado reproductor & $\begin{array}{l}\text { Mismo establo } \\
\text { Mismo estado } \\
\text { Extranjero }\end{array}$ & $\begin{array}{l}751 \\
512 \\
395\end{array}$ & $\begin{array}{l}266 \\
219 \\
184\end{array}$ & $\begin{array}{l}35.4 \\
42.7 \\
46.5\end{array}$ \\
\hline
\end{tabular}

\section{Análisis estadístico}

Se estimó la prevalencia general de la parasitosis para cada grupo de edad, así como para cada variable y categoría de los factores de riesgo hipotéticos. Para establecer el riesgo de infección por Cryptosporidium spp., se desarrolló un análisis de regresión logística (Hosmer et al., 2013), en donde la variable dependiente fue el estado de infección parasitario. La selección de las variables independientes se llevó a cabo por el método backward step by step; por ello, según la estadística en la prueba de $\mathrm{Chi}^{2}$, se excluyeron las variables no significativas $(p<0.05)$. Los Odds Ratio (OR) se estimaron para las variables independientes que mostraron significancia estadística en el análisis multivariado $(p<0.05)$. El análisis se desarrolló con el programa Statistics Data Analysis (STATA) v. 9.1

\section{Resultados y discusión}

La prevalencia general a la infección por Cryptosporidium spp. en la población bajo estudio fue de 40\% (669/1658). El Cuadro 1 muestra la prevalencia para cada categoría con las diferentes variables. 
Cuadro 2. Factores de riesgo a la infección por Cryptosporidium spp. identificados en tres establos lecheros de Aguascalientes, México.

\begin{tabular}{|c|c|c|c|c|}
\hline Variable & Categoría & OR & $\begin{array}{c}\text { Intervalo de confianza } \\
(95 \%)\end{array}$ & Valor de p* \\
\hline \multirow{2}{*}{ Edad } & $\begin{array}{c}\text { Grupo de becerras de } 8 \text { a } 14 \\
\text { días de edad }\end{array}$ & 15.2 & $11.2-20.6$ & 0.000 \\
\hline & $\begin{array}{l}\text { Grupo de becerras de } 15 \text { a } \\
21 \text { días de edad }\end{array}$ & 2.5 & $1.9-3.2$ & 0.000 \\
\hline \multirow{2}{*}{$\begin{array}{l}\text { Persona que atiende el nacimiento } \\
\text { de la cría }\end{array}$} & El velador & 2.5 & $1.6-3.9$ & 0.000 \\
\hline & El trabajador del área de recría & 1.7 & $1.2-2.5$ & 0.001 \\
\hline $\begin{array}{l}\text { Nivel de inmunoglobulinas } \\
\text { en las becerras }\end{array}$ & Nivel bajo & 1.85 & $1.1-2.9$ & 0.009 \\
\hline $\begin{array}{l}\text { Tipo e higienede la cama } \\
\text { y/o piso del alojamiento }\end{array}$ & $\begin{array}{l}\text { Cama de aserrín con } \\
\text { reemplazo cada } 5 \text { días }\end{array}$ & 1.8 & $1.1-2.8$ & 0.011 \\
\hline $\begin{array}{l}\text { Tipo de recipiente para tomar } \\
\text { leche o sustituto de leche }\end{array}$ & De plástico & 1.4 & $1.1-1.7$ & 0.000 \\
\hline
\end{tabular}

El análisis de riesgos permitió identificar 5 variables como factores de riesgo a la infección por Cryptosporidium spp. La variable "edad de las becerras" fue la más importante, en especial en el grupo de 8 a 14 días de nacidas $(O R=15.2$; 95\% $\mathrm{Cl} 11.2$ - 20.6; $\mathrm{p}<0.000$ ), seguido por el grupo de becerras de 15 a 21 días de edad $(\mathrm{OR}=2.5 ; 95 \% \mathrm{Cl} 1.9-3.2, \mathrm{p}<0.000)$. Las otras variables identificadas como factores de riesgo fueron: la persona que atiende el nacimiento de la cría, el nivel de inmunoglobulinas, el tipo e higiene de la cama o piso del alojamiento y el tipo de recipiente para tomar leche o sustituto de leche. En el Cuadro 2 se observan las categorías identificadas como factores de riesgo. Ninguna de las variables relacionadas con la operación y administración de los establos mostró ser factor de riesgo. En la variable "persona que atiende el nacimiento de la cría", al técnico de guardia se le reconoció como un factor de protección ( $\mathrm{OR}=0.73 ; 95 \%$ Cl 0.5828 - 9237, $\mathrm{p}<0.008)$.

En este estudio, la prevalencia general de la infección por Cryptosporidium spp. en becerras lactantes ( $\leq 28$ días de edad) fue de $40 \%$, mientras que en una investigación desarrollada en el mismo Estado, que incluyó animales de 8 a 14 días de edad, se encontró 75\% de prevalencia (Castillo et al., 2009). La amplia presencia del parásito en los establos lecheros de Aguascalientes es evidente. Una situación parecida se ha observado en la región central de México (Hidalgo, Jalisco y Estado de México), así como en el estado de Veracruz, en donde otros autores han reportado prevalencias generales de $25,35.7$ y $73.6 \%$, respectivamente, y encontraron animales positivos en al menos 90\% de las unidades de producción estudiadas (Maldonado et al., 1998; Castelan-Hernández et al., 2011; Cano-Romero et al., 2011). Sin embargo, en México las implicaciones en la salud pública a causa de la transmisión zoonótica son inciertas, debido a que existen sólo algunos trabajos sobre la criptosporidiosis en humanos (Valenzuela et al., 2014).

Diversas investigaciones sobre la prevalencia de la criptosporidiosis en becerras lactantes se han desarrollado en regiones ganaderas de otros países, donde se han utilizado diferentes técnicas de diagnóstico, con resultados muy variados, cuyo rango de prevalencia varía entre el 17\% y el 47.9\% (Lefay et al., 2000; Castro-Hermida et al., 2002a Joachim et al., 2003; Ortolani y Soares, 2003; Santín et al., 2004; 
Kváč et al., 2006; Brook et al., 2008; Bhat et al., 2013), lo cual denota la amplia distribución geográfica y elevada frecuencia de este parásito.

La infección por Cryptosporidium spp. en el ganado lechero es más frecuente y de mayor importancia en las becerras lactantes; además, la magnitud de la enfermedad se ve influida por diversos factores como la edad y el estado inmunológico de las becerras, así como por el clima y otros factores relacionados con el manejo durante la crianza. Por consiguiente, la epidemiología de esta parasitosis muestra características particulares en cada unidad y sistema de producción (Fayer et al., 2000).

Las becerras del grupo de 8 a 14 días de edad tuvieron una prevalencia de $81 \%$, la más alta de los cuatro grupos aquí tratados. Este resultado es similar al reportado previamente en Aguascalientes, en el que el mismo grupo de edad tuvo 75\% de tasa de infección (Castillo et al., 2009). En los otros 3 grupos, la prevalencia del parásito estuvo en un rango de 21 a 40\%. En México, se ha encontrado una mayor prevalencia en becerras menores de 4 meses de edad (Cano-Romero et al, 2011; Castelan-Hernández et al., 2011). Y se observa una mayor excreción de ooquistes entre los días 15 y 19 después del nacimiento (Maldonado et al., 1998). Así, la prevalencia en becerras lactantes de acuerdo a la edad es variable; sin embargo, el grupo más afectado es el de los 8 a 15 días de edad (De la Fuente et al., 1999; Ortolani y Soares 2003; Santín et al., 2004, 2008; Feitosa et al., 2004; Avendaño et al., 2010). Esto coincide con el hecho de que en este estudio, el grupo de 8 a 14 días de edad se identificó como el mayor importancia respecto al factor de riesgo por infección de Cryptosporidium spp. ( $O R=15.2)$, seguido por el de 15 a 21 días de edad ( $O R=2.5)$. Otros autores han encontrado resultados similares (Maldonado et al., 1998; De la Fuente et al., 1999; Santín et al., 2004; Trotz - Williams et al., 2007; Brook et al., 2008), lo que indica que el rango de edad de 8 a 21 días es el de mayor riesgo a la infección y a la excreción de ooquistes del parásito; al incrementarse la edad disminuye el riesgo (Maldonado et al, 1998; Santín et al., 2004, 2008), como también se observa en este análisis.

Entonces, se infiere que la contaminación por Cryptosporidium spp. se realiza después de que las becerras entran en contacto con otros animales, con el alimento iniciador y con el agua para beber. Como señalan Maldonado et al. (1998), el alimento iniciador, que roedores o aves contaminan, actúa como fómite y lo convierte en factor de riesgo; asimismo, el agua para beber se ha identificado como un riesgo debido a la contaminación de los ductos del líquido (Sischo et al., 2000) o del recipiente de bebida (Almeida et al., 2010). La edad debe considerarse como un indicador de riesgo por tratarse de un rasgo individual (Silva Ayçaguer, 2005).

La variable "persona que atiende los nacimientos de las crías" se registra como factor de riesgo cuando el velador o el trabajador del área de recría atiende los nacimientos. En los casos donde el técnico de guardia $(O R=0.73)$ se encarga de los partos, esta variable no es un factor de riesgo y contribuye con la protección, porque el trabajo principal del técnico de guardia es precisamente la atención de los nacimientos por las noches y días festivos. De igual forma, cuando en las mismas condiciones, el trabajador del área o el personal de vigilancia se ocupa de los partos, se deduce que la atención no es la adecuada, ya que, además de atender un nacimiento, se les asignan otras actividades. En la literatura no se encontró información sobre este tópico.

En cuanto al tipo e higiene de la cama y del piso del alojamiento: la cama de aserrín, que se cambia cada 5 días, se determinó como factor de riesgo $(O R=1.80)$, 
debido a las características físicas del material, pues en él se acumula humedad y excremento, lo que constituye un medio ideal para que los ooquistes del parásito sobrevivan y contaminen el material. En contraste, Maldonado et al. (1998) y Mohammed et al. (1999) no encontraron relación significativa entre la parasitosis y la cama de desechos de madera. En este contexto, parece ser que independientemente del tipo de cama, influye más la frecuencia de aseo, puesto que dejar por grandes espacios de tiempo las heces, favorece el desarrollo del parásito y las posibilidades de que la becerra o sus alimentos tengan contacto con el material contaminado. De igual forma, los alojamientos con suelo de rejilla y corrales con cama de arena también se han mostrado como factores de riesgo (Muhid et al., 2011 ). En ambos casos, la presencia de desechos de materia fecal, como ocurrió en esta exploración, representan una fuente de contaminación de ooquistes.

Con referencia al tipo de recipiente utilizado para suministrar a las becerras leche o sustituto de leche, se encontró que el material plástico es un factor de riesgo $(O R=1.4)$, lo que más bien podría estar relacionado con su lavado deficiente, porque el material plástico se puede salpicar con heces diarreicas probablemente contaminadas con el parásito y entonces las becerras se infectarán al lamer los recipientes. Esto es factible, pues aunque los alojamientos para becerras en los establos de estudio son individuales, no ofrecen un total aislamiento, por lo que las becerras pueden lamer los recipientes de los alojamientos vecinos en cualquier momento. A este respecto, Almeida et al. (2010) reportan una asociación significativa entre la bandeja de suministro de agua y el riesgo a la infección por C. parvum; los investigadores mencionan que, cuando el líquido se contamina con materia fecal se convierte en un importante reservorio de los ooquistes del protozoario. Sin embargo, Maldonado et al. (1998) argumentaron que ningún tipo de recipiente o biberón son riesgo de infección por Cryptosporidium.

La prevalencia a la infección fue más alta en animales que se vacunaron contra enfermedades diarreicas. Trotz-Williams et al. (2008) encontraron un resultado similar tanto en vacas como en becerras que habían recibido tal profilaxis. De esta forma, la vacunación se percibe como un factor de riesgo para la infección por Cryptosporidum. Sin embargo, Trotz-Williams et al. (2008) argumentan que la vacunación no es un factor de riesgo porque las vacunas comercializadas para prevenir la diarrea en las becerras, y que no son efectivas contra la infección por Cryptosporidium, no tienen un efecto conocido en el riesgo de criptosporidiosis. La vacunación es, más bien, una medida adoptada en respuesta a una alta frecuencia de diarreas en las becerras.

El análisis del nivel de inmunoglobulinas como factor de riesgo se realizó fuera del modelo general, debido a que la prueba se desarrolló sólo en becerras de 1 a 7 días de edad. De esas becerras, 61.7\% presentó un nivel insuficiente de inmunoglobulinas, por lo que esto se consideró un factor de riesgo $(O R=1.85)$. No fue posible establecer la relación entre el nivel de inmunoglobulinas de las becerras y el número de parto de la madre, a pesar de que existen evidencias de que el número de partos influye en la concentración de inmunoglobulina $\mathrm{G}$ en el calostro (Kehoe et al., 2011 ). Trotz et al. (2007) evaluaron el nivel de inmunoglobulinas en becerras lactantes y encontraron valores de proteínas en el suero menores de $5.2 \mathrm{~g} / \mathrm{dL}$, los cuales se relacionaron significativamente con la infección por Cryptosporidium spp. Es bien sabido que los recién nacidos necesitan ingerir calostro antes de transcurridas seis horas para garantizar la absorción intestinal de las inmunoglobulinas; 
además, cantidades séricas de anticuerpos menores a $10 \mathrm{mg} / \mathrm{mL}$ pueden causar hasta 60\% de mortalidad por infecciones gastrointestinales (Weaver et al., 2000).

Aparentemente, los resultados de este estudio sugieren que en las becerras examinadas, el proceso de alimentación con calostro careció de efectividad para protegerlas contra la infección por Cryptosporidium spp. La información obtenida de los establos indicaba que las crías consumían 4 L o más de calostro, pero ninguno de ellos mostró datos que probaran que la primera toma de calostro ocurría dentro de las primeras seis horas después del nacimiento. Por lo tanto, se infirieron deficiencias en el proceso de alimentación posnatal, lo que explicaría la inefectividad de la alimentación con calostro para proteger a las becerras de la infección por Cryptosporidium spp.

Finalmente, es preciso señalar que, a pesar de estos hallazgos, este estudio se llevó a cabo únicamente en 3 establos; por lo tanto, estos resultados no pueden generalizarse a otros establos de México.

\section{Conclusiones}

En este estudio la edad de las becerras se identificó como un factor de riesgo para adquirir la infección por Cryptosporidium spp.: la infección se asoció al grupo de edad de entre 8 y 14 días.

Debe haber personal exclusivamente dedicado a la atención de los nacimientos y el cuidado de las becerras durante el parto y la crianza, de manera que se tenga un mejor control sobre la óptima alimentación con calostro. Esto podría asegurar un adecuado nivel de inmunoglobulinas, que protegería a las becerras de la infección por el parásito y otras enfermedades.

Los resultados obtenidos sugieren extremar la limpieza de los alojamientos individuales, en especial cuando se utilice cama de aserrín; se deberá retirar cualquier resto de excremento y humedad constantemente para evitar la proliferación del parásito. También los recipientes para beber leche o sustituto de leche se habrán de lavar muy bien para prevenir el riesgo a la infección, pues parece más importante la forma y frecuencia del aseo en las instalaciones y equipo que los materiales utilizados.

\section{Financiamiento}

Este proyecto fue financiado por DGEST-SEP (755.05-P).

\section{Agradecimientos}

Los autores agradecen a los ganaderos que participaron en el estudio toda la ayuda otorgada para la realización del mismo.

\section{Conflictos de interés}

Los autores estipulan que no tienen conflictos de interés. 


\section{Contribución de los autores}

Dolores García Romo: diseño del trabajo; muestreo de campo; pruebas de laboratorio; escritura del artículo.

Carlos Cruz Vázquez: coordinación del grupo de trabajo; diseño del estudio; análisis epidemiológico; redacción del artículo.

Teódulo Quezada Tristán: trabajo de campo; análisis estadístico.

Enrique Silva Peña: trabajo de campo y de laboratorio.

Arturo Valdivia Flores: trabajo de campo y de laboratorio.

Sonia Vázquez Flores: pruebas parasicológicas.

Miguel Ramos Parra: análisis de riesgos.

\section{Referencias}

1) Almeida, A.J., Oliveira, F.C.R., Flores, V.M.Q. and Lopes, C.W.G. 2010. Risk factors associated with the occurrence of Cryptosporidium parvum infection in calves. Arquivo Brasileiro de Medicina Veterinária e Zootecnia. 62 (6): 1325-1330.

2) Avendaño, C., Quílez, J. and Sánchez-Acedo, C. 2010. Prevalence of Cryptosporidium in calves in the Ubaté-Chiquinquirá Valley (Colombia). Revista UDCA Actualidad y Divulgación Científica. 13 (1): 41-47.

3) Bhat, S.A., Juyal, P.D., Singh, N.K. and Singla, L.D. 2013. Coprological investigation on neonatal bovine cryptosporidiosis in Ludhiana, Punjab. Journal of Parasitic Diseases. 37 (1): 114-117.

4) Brook, E., Hart, C.A., French, N. and Christley, R. 2008. Prevalence and risk factors for Cryptosporidium spp. infection in young calves. Veterinary Parasitology. 152 (1): 46-52.

5) Cacciò, S.M. and Pozio, E. 2006. Advances in the epidemiology, diagnosis and treatment of cryptosporidiosis. Expert Review of Anti-Infective Therapy. 4 (3): 429-443.

6) Cano-Romero, P., Alonso-Díaz, M.A., Figueroa-Castillo, J.A. and Trigo-Tavera, J.F. 2011. Prevalence and incidence of Cryptosporidium spp. in calves from the central region of Veracruz, Mexico. Tropical and Subtropical Agroecosystems. 13: 567-571.

7) Castelan-Hernández, O.O., Romero-Salas, D., García-Vázquez, Z., Cruz-Vázquez, C., Aguilar-Domínguez, M., Ibarra-Priego, N.D.J. and Muñoz-Melgarejo, S. 2011. Prevalence of bovine cryptosporidiosis in three ecological regions from the central region of Veracruz, México. Tropical and Subtropical Agroecosystems. 13: 461-467.

8) Castillo-García, C., Cruz-Vázquez, C., López-Revilla, R., Sánchez-Garza, M., Rosario-Cruz, R., Vitela, M.I. and Medina-Esparza, L. 2009. Frecuencia e identificación molecular de Cryptosporidium spp. en becerras lactantes mantenidas en confinamiento en Aguascalientes, México. Técnica Pecuaria en México. 47 (4): 425-434.

9) Castro-Hermida, J., González, L.Y., Mezo, M.M. and Ares, M.E. 2002a. A study of cryptosporidiosis in a cohort of neonatal calves. Veterinary Parasitology. 106 (1): 11-17.

10) Castro-Hermida, J., González, L.Y. and Ares, M.E. 2002b. Prevalence and risk factors involved in the spread of neonatal bovine cryptosporidiosis in Galicia (NW Spain). Veterinary Parasitology. 106 (1): 1-10. 
11) Castro-Hermida, J., Carro-Corral, C., González-Warleta, M. and Mezo, M. 2006. Prevalence and intensity of infection of Cryptosporidium spp. and Giardia duodenalis in dairy cattle in Galicia (NW Spain). Journal of Veterinary Medicine Series B. 53 (5): 244-246.

12) De la Fuente, R., Luzón, M., Ruiz-Santa-Quiteria, J.A., García, A., Cid, D., Orden, J.A., García, S., Sanz, R. and Gómez-Bautista, M. 1999. Cryptosporidium and concurrent infections with other major enterophatogens in 1 to 30-day-old diarrheic dairy calves in central Spain. Veterinary Parasitology. 80 (3): 179-185.

13) Fayer, R., Morgan, U. and Upton, J.S. 2000. Epidemiology of Cryptosporidium: transmission, detection and identification. International Journal for Parasitology. 30 (13): 1305-1322.

14) Fayer, R., Santín, M. and Xiao, L. 2005. Cryptosporidium bovis n. sp. (Apicomplexa: Cryptosporidiidae) in cattle (Bos taurus). Journal of Parasitology. 91 (3): 624-629.

15) Fayer, R., Santín, M. and Trout, J.M. 2007. Prevalence of Cryptosporidium species and genotypes in mature dairy cattle on farms in eastern United States compared with younger cattle from the same locations. Veterinary Parasitology. 145 (3-4): 260-266.

16) Fayer, R., Santín, M. and Trout, J.M. 2008. Cryptosporidium ryanae n. sp. (Apicomplexa: Cryptosporidiidae) in cattle (Bos taurus). Veterinary Parasitology. 156 (3): 191-198.

17) Feitosa, F.L.F., Shimamura, G.M., Roberto, T., Meireles, M.V., Nunes, C.M., Ciarlini, P.C. and Borges, A.S. 2004. Prevalence of cryptosporidiosis in calves from Araçatuba region, São Paulo State, Brazil. Ciencia Rural. 34 (1): 189-193.

18) Hosmer, D.W., Lemeshow, S. and Sturdivant, R.X. 2013. Applied Logistic Regression. 3th edition. New Jersey: Wiley.

19) Joachim, A., Krull, T., Schwarzkopf, J. and Daugachies, A. 2003. Prevalence and control of bovine cryptosporidiosis in German dairy herds. Veterinary Parasitology. 112 (4): 277-278.

20) Kehoe, S.I., Heinrichs, A.J., Moody, M.L, Jones, C.M. and Long, M.R. 2011. Comparison of immunoglobulin $G$ concentrations in primiparous and multiparous bovine colostrum. Professional Animal Scientist. 27 (3): 176-180.

21) Kváč, M., Kouba, M. and Vítovec, J. 2006. Age related and housing dependence of Cryptosporidium infection of calves from dairy and beef herds in South Bohemia, Czech Republic. Veterinary Parasitology. 137 (3-4): 202-209.

22) Lefay D., Naciri, M., Poirer, P. and Chermette, R. 2000. Prevalence of Cryptosporidium infection in calves France. Veterinary Parasitology. 89 (1): 1-9.

23) Lindsay, D.S., Upton, S.J., Owens, D.S., Morgan, U.M., Mead, J.R. and Blagburn, B.L. 2000. Cryptosporidium andersoni n. sp. (Apicomplexa: Cryptosporiidae) from cattle, Bos taurus. Journal of Eukaryotic Microbiology. 47(1): 91-95.

24) Maldonado, C.S., Atwill, E.R., Saltijeral, O.J.A. and Herrera, A.L.C. 1998. Prevalence of and risk factors for shedding of Cryptosporidium parvum in Holstein Freisian dairy calves in Central México. Preventive Veterinary Medicine. 36 (29): 95-107.

25) Martínez, R.A. and Ortega, SJ. 2011 . Manual de Laboratorio de Inmunología Básica y Clínica. Universidad Autónoma Chapingo: México.

26) Mohammed, H.O., Wade, S.E., and Schaaf, S. 1999. Risk factors associated with Cryptosporidium parvum infection in dairy cattle in southeastern New York State. Veterinary Parasitology. 83 (1): 1-13. 
27) Muhid, A., Robertson, I., Ng, J.S.Y., and Ryan, U. 2011. Prevalence of and management factors contributing to Cryptosporidium sp. infection in pre-weaned and postweaned calves in Johor, Malaysia. Experimental Parasitology. 127 (2): 534-538.

28) Ng, J.S.Y., Eastwood, K., Walker, B., Durrheim, D.N., Massey, P.D., Porigneaux, P. and Ryan, U. 2012. Evidence of Cryptosporidium transmission between cattle and humans in northern New South Wales. Experimental Parasitology. 130 (4): 437-441.

29) Ortolani, E.R. and Soares, P.C. 2003. Aspectos epidemiológicos de la criptosporidiosis en becerros de rebaños lecheros. Parasitología Latinoamericana. 58 (3-4): 122-127.

30) Ramírez, N.E., Ward, L.A. and Sreevatsan S. 2004. A review of biology and epidemiology of cryptosporidiosis in humans and animals. Microbes and Infection. 6 (8): 773-785.

31) Santín, M., Trout. J.M., Xiao, L., Zhou, L., Greiner, E. and Fayer, R. 2004. Prevalence and age-related variation of Cryptosporidium species and genotypes in dairy calves. Veterinary Parasitology. 122 (2): 103-117.

32) Santín, M., Trout, J.M. and Fayer, R. 2008. A longitudinal study of cryptosporidiosis in dairy cattle from birth to 2 years of age. Veterinary Parasitology. 155 (1): 15-23.

33) Silva-Ayçaguer, L.C. 2005. Una ceremonia estadística para identificar factores de riesgo. Salud colectiva. 1(3): 309-322.

34) Silverlås, C. and Blanco-Penedo, I. 2013. Cryptosporidium spp. in calves and cows from organic and conventional dairy herds. Epidemiology and Infection. 141 (3): 529-539.

35) Sischo, W.M., Atwill, E.R., Lanyon, L.E. and George, J. 2000. Cryptosporidia on dairy farms and the role these farms may have in contaminating surface water supplies in the northeastern United States. Preventive Veterinary Medicine. 43 (4): 253-267.

36) Starkey, S.R., Kimber, K.R., Wade, S.E., Schaaf, S.L., White, M.E. and Mohammed, H.O. 2006. Risk Factors Associated with Cryptosporidium Infection on dairy farms in New York State Watershed. Journal Dairy Science. 89 (11): 4229-4236.

37) Trotz-Williams, L.A., Brenna, D.J., Wayne, M.S., Kenneth, E.L. and Peregrine, S.A. 2005. Prevalence of Cryptosporidium parvum infection in southwestern Ontario and its association with diarrhea in neonatal dairy calves. Canadian Veterinary Journal. 46 (4): 349-351.

38) Trotz-Williams, L.A., Wayne-Martin, S., Leslie, K.E., Duffield, T., Nydam, D.V. and Peregrine, A.S. 2007. Calf-level risk factors for neonatal diarrhea and shedding of Cryptosporidium parvum in Ontario dairy calves. Preventive Veterinary Medicine. 82 (1): 12-28.

39) Trotz-Williams, L.A., Wayne-Martin, S., Leslie, K.E., Duffield, T., Nydam, D.V. and Peregrine, A.S. 2008. Association between management practices and within-herd prevalence of Cryptosporidium parvum shedding on dairy farms in southern Ontario. Preventive Veterinary Medicine. 83 (1): 11-23.

40) Uga, S., Matsuo, J., Kono, E., Kimura, K., Inoue, M., Rai, S.K. and Ono, K. 2000. Prevalence of Cryptosporidium parvum infection and pattern oocysts sheding in calves in Japan. Veterinary Parasitology. 94 (1): 27-32. 
41) Valenzuela, O., González-Díaz, M., Garibay-Escobar, A., Burgara-Estrella, A., Cano, M., Durazo, M., Bernal, R.M., Hernández, J., Xiao, L. 2014. Molecular characterization of Cryptosporidium spp. in children from Mexico. PloS One. 9 (4): e96128.

42) Weaver, D.M., Tyler, J.W., VanMetre, D.C., Hostetler, D.E. and Barrington, G.M. 2000. Passive transfer of colostral immunoglobulins in calves. Journal of Veterinary Internal Medicine. 14(6): 569-577. 